\title{
Biodegradable lubricating mesoporous silica nanoparticles for osteoarthritis therapy
}

\author{
Li WAN ${ }^{1,2, \uparrow}$, Yi WANG ${ }^{1, \uparrow}$, Xiaolong TAN ${ }^{1}$, Yulong SUN ${ }^{1}$, Jing LUO ${ }^{3}$, Hongyu ZHANG ${ }^{1, *}$ \\ ${ }^{1}$ State Key Laboratory of Tribology, Department of Mechanical Engineering, Tsinghua University, Beijing 100084, China \\ ${ }^{2}$ College of Mining, Guizhou University, Guiyang 550025, China \\ ${ }^{3}$ Beijing Research Institute of Automation for Machinery Industry Co., Ltd., Beijing 100120, China \\ Received: 22 January 2020 / Revised: 12 March 2020 / Accepted: 27 March 2020 \\ (C) The author(s) 2020.
}

\begin{abstract}
Osteoarthritis is characterized by lubrication failure of the articular cartilage and severe inflammation of the joint capsule. Lubricating mesoporous silica nanoparticles (MSNs) have been developed for the treatment of osteoarthritis based on enhanced lubrication and local drug delivery. However, MSNs are difficult to degrade in vivo in a short time, resulting in potential toxic effect due to bioaccumulation. In this study, biodegradable MSNs (bMSNs) were prepared through an oil-water biphase stratification method, and modified with poly(2-methacryloyloxyethyl phosphocholine) (PMPC) to synthesize lubricating drug-loaded nanoparticles (bMSNs- $\mathrm{NH}_{2} @ \mathrm{PMPC}$ ) by photopolymerization. The in vitro degradation test demonstrated that the bMSNs and bMSNs- $\mathrm{NH}_{2} @ \mathrm{PMPC}$ almost degraded within 7 days. The tribiological test showed that the lubrication property of the bMSNs- $\mathrm{NH}_{2} @ \mathrm{PMPC}$ was greatly improved, with a reduction of $50 \%$ in the friction coefficient (COF) compared with the bMSNs. It was attributed to hydration lubrication mechanism by which a tenacious hydration layer is formed surrounding the zwitterionic headgroups $\left(\mathrm{N}^{+}\left(\mathrm{CH}_{3}\right)_{3}\right.$ and $\left.\mathrm{PO}_{4}^{-}\right)$in $\mathrm{PMPC}$ polyelectrolyte polymer. Additionally, the bMSNs- $\mathrm{NH}_{2} @ \mathrm{PMPC}$ maintained excellent lubrication property under degradation and achieved sustained drug release behavior compared with the bMSNs. In summary, the biodegradable bMSNs- $\mathrm{NH}_{2} @$ PMPC developed in this study with the properties of enhanced lubrication and drug delivery may be a promising approach for osteoarthritis therapy.
\end{abstract}

Keywords: biodegradable; mesoporous silica nanoparticles; lubrication; drug delivery; osteoarthritis

\section{Introduction}

Osteoarthritis is a chronic joint disease characterized by articular cartilage degeneration and inflammation of joint capsule, which severely affects quality of life for the patients [1,2]. Currently, the clinical treatment methods mainly include intra-articular injection of viscosupplement (hyaluronic acid), oral administration of chondroprotective agent (glucosamine), and surgical replacement (joint arthroplasty) according to the symptoms of osteoarthritis [3-5]. Although surgical replacement can eliminate or alleviate joint pain immediately, it may result in serious complications by aggravating the burden of other organs, especially when patients have symptomatic diseases, such as high blood pressure, heart disease, and diabetes [6, 7]. Consequently, it is more preferrable to treat osteoarthritis at an early stage. As the occurrence of osteoarthritis is highly related with the significant increase in joint friction and the stimulation in inflammatory response, a therapy combining lubrication restoration and drug intervention is considered effective as an innovative strategy for the treatment of osteoarthritis $[8,9]$.

Recently, lubricating hollow silica nanoparticles

$\dagger$ These authors contributed equally to this work.

* Corresponding author: Hongyu ZHANG, E-mail: zhanghyu@tsinghua.edu.cn 
and mesoporous silica nanoparticles (MSNs) have been developed to treat osteoarthritis [10-12]. These nanoparticles could not only greatly enhance lubrication but also show chondroprotective potential through encapsulating anti-inflammatory drugs into the mesoporous channels. The synergistic effect of lubrication enhancement and sustained drug release of the nanoparticles has made a significant progress for the treatment of early-stage osteoarthritis. However, the serious long term bioaccumulation of the nanoparticles is neglected in these studies. Since the "-Si-O-Si-" skeleton structure is stable, the biodegradability of MSNs is very slow in vivo, which may be extremely aggregated in vital organs of human body, such as liver, spleen, and bladder [13]. Subsequently, the long term bioaccumulation of the nanoparticles would result in serious problems such as inflammatory reaction, oxidative damage, and organ fibrosis [14, 15]. The concern of accumulation toxicity of the MSNs has greatly hindered the progression to clinical transformation $[16,17]$. Therefore, it is considered necessary to develop biodegradable MSNs (bMSNs), which not only retain the advantage of drug release performance but also rapidly degrade and metabolize in organisms [18-22].

In the present study, bMSNs are prepared through oil-water biphase stratification method. As shown in Fig. 1(b), tetraethyl orthosilicate (TEOS) in the upper oil phase continuously penetrates into the aqueous phase and gradually hydrolyzes on the "Seed" surface, eventually forming bMSNs with dendritic channels. Compared with the dense MSNs prepared by rapid condensing template method as shown in Fig. 1(a), bMSNs have a loose "-Si-O-Si-" skeleton structure, and thus biodegrade rapidly in vivo. Subsequently, the bMSNs are grafted with poly(2-methacryloyloxyethyl phosphocholine) (PMPC) to synthesize lubricating drug-loaded nanoparticles (bMSNs- $\mathrm{NH}_{2} @ \mathrm{PMPC}$ ) by photopolymerization. As indicated in Fig. 1(c), the surface of bMSNs with hydroxyl group is modified with amino group and photoinitiator (I2959-Tos), and reacted with the MPC monomer to obtain bMSNs$\mathrm{NH}_{2} @$ PMPC under UV-irradiation. It is hypothesized
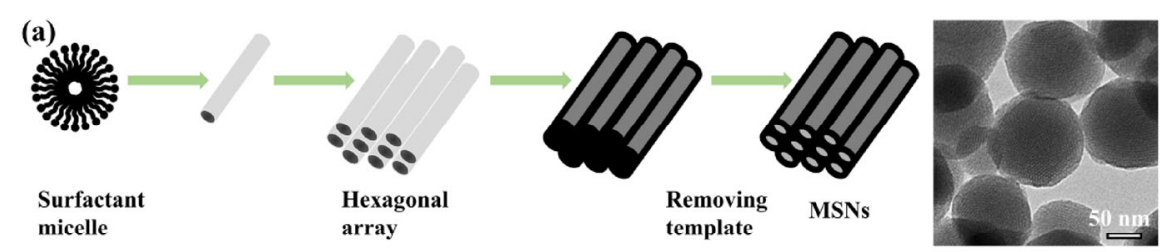

(b)
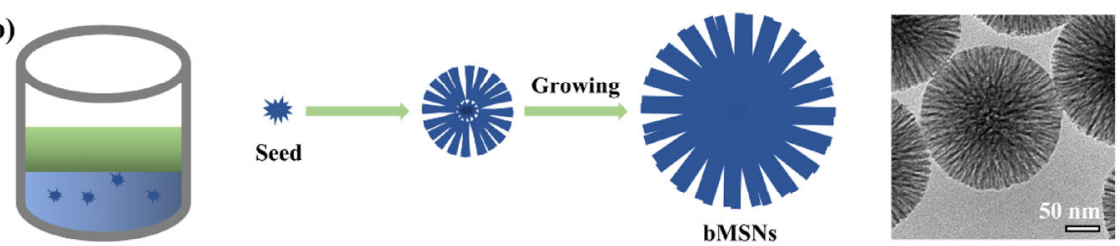

(c)

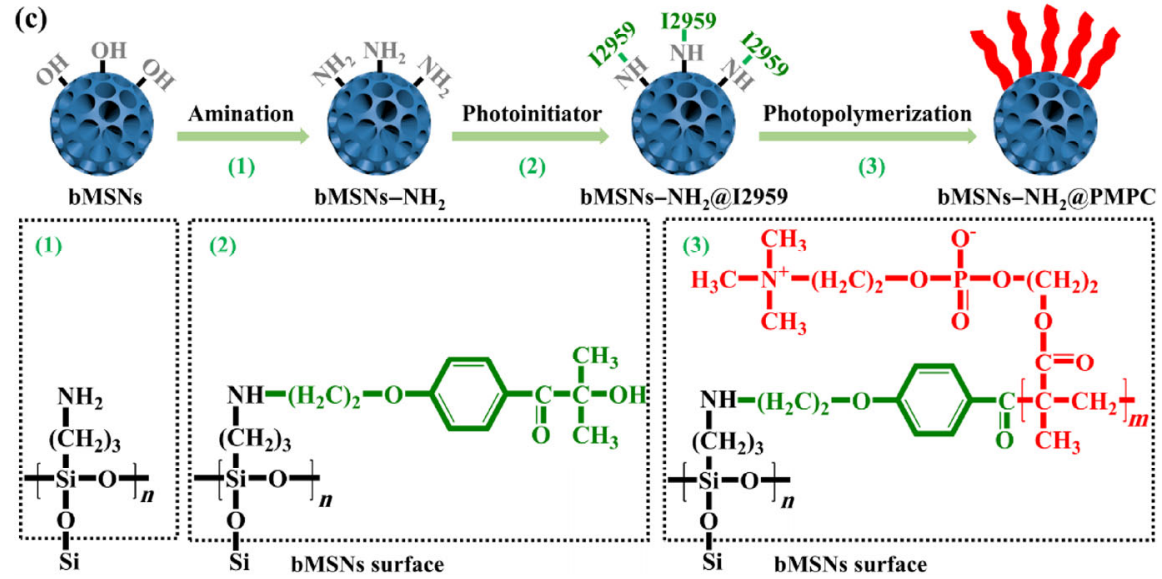

Fig. 1 Schematic illustration showing the synthesis of nanoparticles: (a) MSNs by rapid condensing template method, (b) bMSNs by oil-water biphase stratification method, and (c) bMSNs- $\mathrm{NH}_{2} @ \mathrm{PMPC}$ by photopolymerization. 
that the bMSNs- $\mathrm{NH}_{2} @ \mathrm{PMPC}$ with the properties of biodegradability, enhanced lubrication, and drug delivery can be used as a promising agent in biomedical applications, e.g., the treatment of osteoarthritis.

\section{Materials and methods}

\subsection{Materials and reagents}

2-methacryloyloxyethyl phosphocholine (MPC, 99\%) was purchased from Joy-Nature Corp., Nanjing, China. Cetyltrimethylammonium chloride solution (CTAC, $25 \mathrm{wt} \%$ ), tetraethyl orthosilicate (TEOS, 98\%), cetyltrimethylammonium bromide (CTAB, 98\%), p-toluenesulfonyl chloride ( $\mathrm{TsCl}, 99 \%)$, and (3aminopropyl) triethoxysilane (APTES, 98\%) were purchased from J\&K Scientific Co., Beijing, China. Rhodamine B (RhB, 98\%), 2-hydroxy-4'-(2hydroxyethoxy)-2-methylpropiophenone (I2959, 96\%), and simulated body fluid (SBF) were purchased from Solarbio Co., Beijing, China. Toluene, triethanolamine (TEA), N,N-dimethylformamide (DMF), dichloromethane, methanol, cyclohexane, and other reagents were obtained from Beijing Chemical Reagent Co., Beijing, China.

\subsection{Synthesis of MSNs}

$0.14 \mathrm{~g}$ of $\mathrm{NaOH}, 0.5 \mathrm{~g}$ of $\mathrm{CTAB}$, and $240 \mathrm{~mL}$ of deionized water were stirred at $80{ }^{\circ} \mathrm{C}$ for $30 \mathrm{~min}$. Subsequently, $5 \mathrm{~mL}$ of TEOS was slowly added and stirred for $6 \mathrm{~h}$. The precipitate was gathered through filtration, and washed thrice with deionized water and methanol. Then, it was uniformly stirred in an acidic methanol solution (containing $150 \mathrm{~mL}$ of methanol and $1.5 \mathrm{~mL}$ of $10 \mathrm{~mol} / \mathrm{L} \mathrm{HCl}$ ) at $60{ }^{\circ} \mathrm{C}$ for $12 \mathrm{~h}$ to remove the template. The final product (MSNs) was filtered, washed sufficiently with methanol, and dried overnight under vacuum [23].

\subsection{Synthesis of bMSNs}

The bMSNs were synthesized using a biphase stratification method [24]. $120 \mathrm{~mL}$ of CTAC, $0.9 \mathrm{~g}$ of TEA, and $180 \mathrm{~mL}$ of deionized water were continuously stirred at $60^{\circ} \mathrm{C}$ for $1 \mathrm{~h}$. Subsequently, a mixed solution ( $5 \mathrm{~mL}$ of TEOS and $95 \mathrm{~mL}$ of cyclohexane, $5 \mathrm{v} / \mathrm{v} \%$ ) was gently added to the aqueous solution to form an environment in which the aqueous phase and the organic phase were layered. The mixture was stirred at a low speed ( $90 \mathrm{rpm}$ ) for $60 \mathrm{~h}$. The bMSNs containing the CTAC template in aqueous phase were collected by centrifugation. Finally, it was uniformly dispersed in the acidic methanol solution, and stirred at $60{ }^{\circ} \mathrm{C}$ for $24 \mathrm{~h}$ to remove the template to obtain bMSNs.

\subsection{Synthesis of aminated bMSNs- $\mathrm{NH}_{2}$}

Briefly, bMSNs $(1 \mathrm{~g})$, anhydrous toluene $(100 \mathrm{~mL})$, and APTES $(10 \mathrm{~mL})$ were refluxed under nitrogen atmosphere at $110{ }^{\circ} \mathrm{C}$ for $24 \mathrm{~h}$. Afterwards, the mixture was centrifuged at $8,000 \mathrm{rpm}$ for $10 \mathrm{~min}$. The product was washed thrice with toluene and methanol, and dried overnight under vacuum to obtain bMSNs- $\mathrm{NH}_{2}$.

\subsection{Synthesis of bMSNs- $\mathrm{NH}_{2} @ \mathrm{PMPC}$}

I2959 (2.69 g, $0.012 \mathrm{~mol}), \mathrm{TsCl}(1.90 \mathrm{~g}, 0.010 \mathrm{~mol}), \mathrm{KOH}$ $(2.24 \mathrm{~g}, 0.040 \mathrm{~mol})$, and $30 \mathrm{~mL}$ of dichloromethane were stirred at $25{ }^{\circ} \mathrm{C}$ for $2 \mathrm{~h}$. Then, the mixture was washed with deionized water, and the organic phase was dried over $\mathrm{Na}_{2} \mathrm{SO}_{4}$ and distilled. Silica gel column chromatography, with an elution of methylene chloride and ethyl acetate $(1: 4, v / v)$, was employed to purify the product (2959-Tos, photoinitiator). Subsequently, I2959-Tos ( $1 \mathrm{~g}), \mathrm{K}_{2} \mathrm{CO}_{3}(2 \mathrm{~g})$, bMSNs- $\mathrm{NH}_{2}$ (400 mg), and $10 \mathrm{~mL}$ of DMF were stirred at $120{ }^{\circ} \mathrm{C}$ for $24 \mathrm{~h}$ under nitrogen atmosphere. The mixture was centrifuged at $8,000 \mathrm{rpm}$ for $10 \mathrm{~min}$. The precipitate was washed sufficiently with ethanol and deionized water, and dried to obtain the product of bMSNs$\mathrm{NH}_{2} @ \mathrm{I} 2959$.

Finally, bMSNs-NH $\mathrm{N}_{2} @ \mathrm{I} 2959$ (200 mg), MPC (1 g), and $10 \mathrm{~mL}$ of deionized water were stirred at $90{ }^{\circ} \mathrm{C}$, and irradiated with ultraviolet light $\left(5 \mathrm{~mW} / \mathrm{cm}^{2}\right)$ under nitrogen atmosphere for $2 \mathrm{~h}$. The precipitate was collected by centrifugation at $8,000 \mathrm{rpm}$ for $30 \mathrm{~min}$, washed sufficiently with deionized water, and finally dried to obtain the product of bMSNs- $\mathrm{NH}_{2} @$ PMPC.

\subsection{Characterizations}

Transmission electron microscopy (TEM) was conducted using an H-7650B instrument (Hitachi, Japan) to examine the morphology of the nanoparticles. Particle size analyzer (Zetasizer Nano ZS, Malvern Instruments, Malvern, UK) was employed to measure the hydro- 
dynamic diameter and zeta potential of the nanoparticles based on dynamic light scattering (DLS). Nitrogen adsorption-desorption isotherm and pore size distribution were implemented using a NOVA4000 equipment (Quantachrome, CA, USA) to obtain the specific surface area and pore volume of the nanoparticles. Additionally, Fourier transform infrared (FTIR) spectrum was recorded employing a Nexus 670 spectrometer (Thermo-Nicolet, Madison, WI, USA) at a wavelength from 500 to $2,500 \mathrm{~cm}^{-1}$. Thermogravimetric analysis (TGA) was performed using a Q5000IR instrument (TA Instruments, New Castle, DE, USA) at a heating rate of $10{ }^{\circ} \mathrm{C} / \mathrm{min}$ from 25 to $800{ }^{\circ} \mathrm{C}$. Scanning electron microscopy (SEM, SU8220, Hitachi, Japan) associated with energy dispersive spectroscopy (EDS) was used to characterize the surface morphology of the wear areas on the samples in the tribological test.

\subsection{In vitro biodegradation}

An acidic SBF was used to test the biodegradation characteristics of the nanoparticles including MSNs, bMSNs, and bMSN-NH $\mathrm{N}_{2} @ \mathrm{PMPC}$. Briefly, an equivalent amount of the nanoparticles $(7.0 \mathrm{mg})$ was placed in $20 \mathrm{~mL}$ of SBF $(\mathrm{pH}=5.5)$ at $37^{\circ} \mathrm{C}$. Aliquots were taken and replaced by equal amount of fresh SBF at regular intervals of 1,3 , and 7 days. The extracted solution was centrifuged at $8,000 \mathrm{rpm}$ to collect the nanoparticles. The morphology of the biodegraded nanoparticles was observed using the TEM.

\subsection{Rheological test}

The rheological performance of the bMSNs- $\mathrm{NH}_{2} @ \mathrm{PMPC}$ nanoparticles under different concentrations $(2,4,6$, and $10 \mathrm{mg} / \mathrm{mL}$ ) in aqueous suspensions was examined using a Physica MCR301 rheometer (Anton Paar, Austria), which was equipped with a cone-and-plate module (diameter: $49.955 \mathrm{~mm}$; cone angle: $0.988^{\circ}$ ). The curves of viscosity versus shear rate $\left(10-8,000 \mathrm{~s}^{-1}\right)$ was obtained by dropping $1 \mathrm{~mL}$ of aqueous suspension on the plate under shearing mode.

\subsection{Tribological test}

The lubrication property of bMSNs and bMSNs$\mathrm{NH}_{2} @$ PMPC nanoparticles in aqueous suspensions (5 mg/mL) was tested using a UMT-3 universal materials tester (Bruker, Billerica, MA, USA) in reciprocating mode (amplitude: $4 \mathrm{~mm}$ ). The upper and lower friction tribopairs were polytetrafluoroethylene (PTFE) sphere (diameter: $8 \mathrm{~mm}$ ) and polished Ti6Al4V sheet. The tribological test was performed at different loads $(1,2$, and $4 \mathrm{~N})$ and frequencies $(1,3$, and $5 \mathrm{~Hz})$, each for a duration of $15 \mathrm{~min}$. The curve of friction coefficient (COF) versus time was recorded for the test. The apparent maximum contact pressure was calculated based on Hertz equation for ball-on-flat configuration $[25,26]$.

$$
P=\left(\frac{1}{\pi}\right) \cdot \sqrt[3]{\frac{6 F}{\left(\frac{1-\mu_{1}^{2}}{E_{1}}+\frac{1-\mu_{2}^{2}}{E_{2}}\right)^{2} R^{2}}}
$$

where $P$ is the contact pressure (MPa), $F$ is the applied load $(1,2$, and $4 \mathrm{~N}), \mu_{1}$ and $\mu_{2}$ are the Poisson's ratio of PTFE (0.3) and Ti6Al4V (0.3), $E_{1}$ and $E_{2}$ are the elastic modulus of PTFE (0.5 GPa) and Ti6Al4V (110 GPa), and $R(4 \mathrm{~mm})$ is the radius of the PTFE sphere. Consequently, $P$ was calculated to be $15.4 \mathrm{MPa}(1 \mathrm{~N})$, 19.3 MPa (2 N), and 24.4 MPa (4 N), respectively.

\subsection{Lubrication property of biodegraded nano- particles}

The lubrication property of biodegraded nanoparticles in aqueous suspensions (bMSNs and bMSNs$\mathrm{NH}_{2} @$ PMPC, $5 \mathrm{mg} / \mathrm{mL}$ ) was investigated employing the UMT-3 universal materials tester. The bMSNs and bMSNs- $\mathrm{NH}_{2} @$ PMPC were dispersed ultrasonically in SBF $(\mathrm{pH}=5.5)$ and cultivated in a shaker at $37^{\circ} \mathrm{C}$. Aliquots were taken and replaced by equal amount of fresh SBF at regular intervals of 1, 3, 5, and 7 days. The aqueous suspension was tested under the following experimental conditions: load: $2 \mathrm{~N}$, frequency: $3 \mathrm{~Hz}$. The other test parameters were the same as above mentioned.

\subsection{In vitro drug loading and release}

$\mathrm{RhB}$ was chosen as a model cargo to test the drug loading and release characteristics of the nanoparticles. Briefly, $20 \mathrm{mg}$ of bMSNs and bMSNs- $\mathrm{NH}_{2} @$ PMPC was added to $10 \mathrm{~mL}$ of $\mathrm{RhB}$ solution $(0.5 \mathrm{mM})$. After stirring at room temperature for $2 \mathrm{~h}$, the precipitate was collected by centrifugation, washed with deionized 
water, and dried under vacuum to obtain RhB-loaded nanoparticles. The amount of $\mathrm{RhB}$ remaining in the solution was calculated by measuring the absorbance of the supernatant at $520 \mathrm{~nm}$ employing a UV-vis spectrophotometer (UV-8000s, Metash Instruments, Shanghai, China). The loading capacity (LC) and encapsulation efficiency (EE) of the nanoparticles were obtained by the following equations:

$$
\mathrm{LC}(\%)_{\mathrm{bMSNs}}=\frac{\text { Amount of loaded RhB }}{\text { Amount of RhB }- \text { Loaded bMSNs }} \times 100
$$

$$
\begin{aligned}
& \mathrm{LC}(\%)_{\mathrm{bMSN}_{-} \mathrm{NH}_{2} @ \mathrm{PMPC}} \\
& =\frac{\text { Amount of loaded RhB }}{\text { A mount of RhB-loaded bMSNs- } \mathrm{NH}_{2} @ \mathrm{PMPC}} \times 100
\end{aligned}
$$

$$
\mathrm{EE}(\%)=\frac{\text { Amount of loaded RhB }}{\text { Amount of added RhB }} \times 100
$$

The drug release of the nanoparticles was tested by the dialysis process. Briefly, $20 \mathrm{mg}$ of drug-loaded nanoparticles including MSNs, bMSNs, and bMSN$\mathrm{NH}_{2} @$ PMPC were ultrasonically dispersed into $10 \mathrm{~mL}$ of phosphate buffer solution (PBS). $2 \mathrm{~mL}$ of the suspension was put into to a dialysis bag with a molecular weight cutoff of 8,000-10,000, and dialyzed in $18 \mathrm{~mL}$ of PBS at $37^{\circ} \mathrm{C}$. Subsequently, $2 \mathrm{~mL}$ of RhBcontaining dialysate was sucked out, and $2 \mathrm{~mL}$ of fresh PBS was added at regular intervals. The absorbance was measured by the spectrophotometer at $520 \mathrm{~nm}$, and the amount of $\mathrm{RhB}$ released from the nanoparticles was calculated. After all the values at regular intervals were obtained, the cumulative release-time curve of RhB was plotted.

\subsection{Statistical analysis}

The data were presented as mean \pm standard deviation (SD), and similar independent tests were repeated at least three times to verify the results. The statistical analysis was performed using GraphPad Prism software (Version 5.0, GraphPad Software Inc., USA).

\section{Results and discussion}

\subsection{Characterizations of nanoparticles}

The morphologies of the MSNs, bMSNs, and bMSNs-
$\mathrm{NH}_{2} @$ PMPC are observed by TEM. Figure 2(a) shows that the average diameter of MSNs is approximately $100 \mathrm{~nm}$, and the parallel channels were contained. Figure 2(b) demonstrates that the average diameter of bMSNs is roughly $240 \mathrm{~nm}$, and internal pores present a radial distribution. Figure 2(c) indicates that the bMSNs- $\mathrm{NH}_{2} @$ PMPC are covered by a polymer layer with a thickness of $\sim 25 \mathrm{~nm}$. The pores of bMSN$\mathrm{NH}_{2} @$ PMPC can be faintly seen due to the presence of the PMPC polymer layer.

Nitrogen adsorption-desorption isotherms are examined to analyze the mesopores of the nanoparticles. As demonstrated in Fig. 2(d), all the isotherms of MSNs, bMSNs, and bMSNs- $\mathrm{NH}_{2} @ \mathrm{PMPC}$ show typical type IV pattern, indicating a mesoporous structure. The specific surface area and pore volume of the nanoparticles were calculated, based on the BrunauerEmmett-Teller (BET) model, as $743.1 \mathrm{~m}^{2} / \mathrm{g}$ and $0.614 \mathrm{~mL} / \mathrm{g}$ for MSNs, and 1,169.6 $\mathrm{m}^{2} / \mathrm{g}$ and $1.485 \mathrm{~mL} / \mathrm{g}$ for bMSNs. These values decrease significantly to be $179.89 \mathrm{~m}^{2} / \mathrm{g}$ and $0.568 \mathrm{~mL} / \mathrm{g}$ for bMSNs-NH $\mathrm{NH}_{2} @ \mathrm{PMPC}$ after grafting of polyelectrolyte polymer on the nanoparticle surface. Pore size distribution of the three nanoparticles, which is obtained by Barrett-JoynerHalenda (BJH) analysis, is shown in Fig. 2(e). It is obvious that the pore size distribution of MSNs is concentrated at $3 \mathrm{~nm}$, while the pore size of bMSNs is distributed from 3 to $12 \mathrm{~nm}$, corresponding to the mesopores from the central core to the outer surface of the nanoparticles. However, the pore size of bMSNs$\mathrm{NH}_{2} @ \mathrm{PMPC}$ is hardly shown due to the presence of the polyelectrolyte polymer.

FTIR spectroscopy analysis of the nanoparticles is shown in Fig. 2(f). The spectra of bMSNs, bMSNs- $\mathrm{NH}_{2}$ bMSNs- $\mathrm{NH}_{2} @ \mathrm{I} 2959$, and bMSNs-NH $\mathrm{NH}_{2} @ \mathrm{PMPC}$ all indicate the absorption band of $\mathrm{Si}-\mathrm{O}-\mathrm{Si}$ at $1,089 \mathrm{~cm}^{-1}$, and only slight differences are noted in the spectrum of bMSNs- $\mathrm{NH}_{2} @ \mathrm{I} 2959$ compared with bMSNs- $\mathrm{NH}_{2}$. The absorption band of $\mathrm{C}=\mathrm{O}$ appears at $1,730 \mathrm{~cm}^{-1}$, and the absorption bands of $\mathrm{P}-\mathrm{O}$ and $\mathrm{P}=\mathrm{O}$ in $\mathrm{PO}_{4}^{-}$ appear at 966 and $1,242 \mathrm{~cm}^{-1}$, as demonstrated in the spectrum of bMSNs- $\mathrm{NH}_{2} @ \mathrm{PMPC}$. The result indicates that PMPC polyelectrolyte polymer has been successfully grafted on the bMSNs surface via photopolymerization.

TGA curves of the nanoparticles are presented in Fig. 2(g), and the data are established with the 

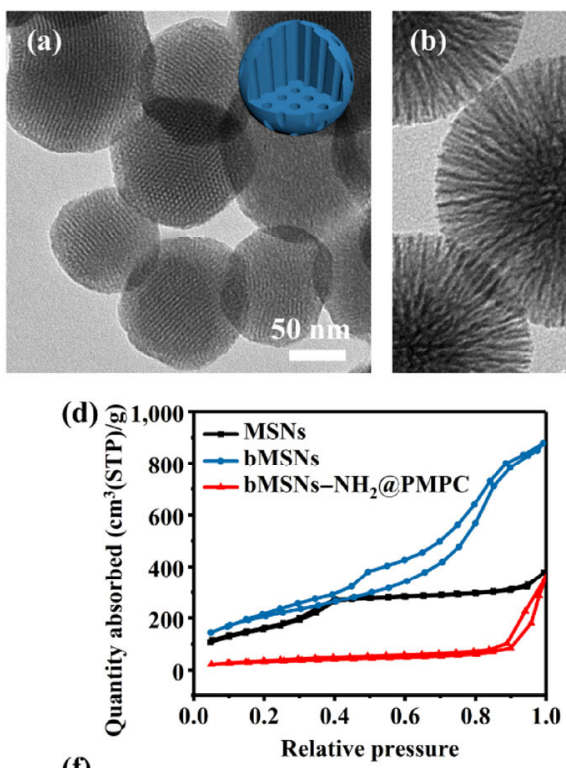

(f)

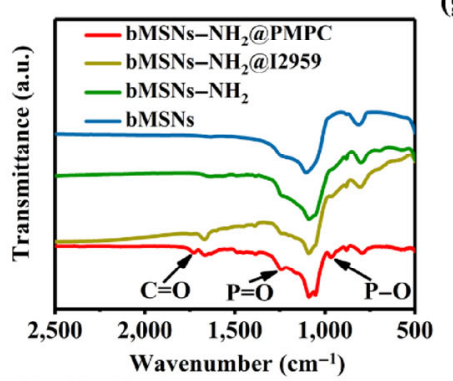

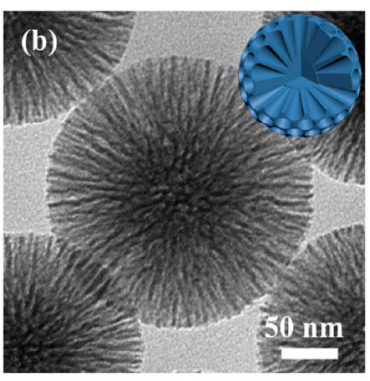
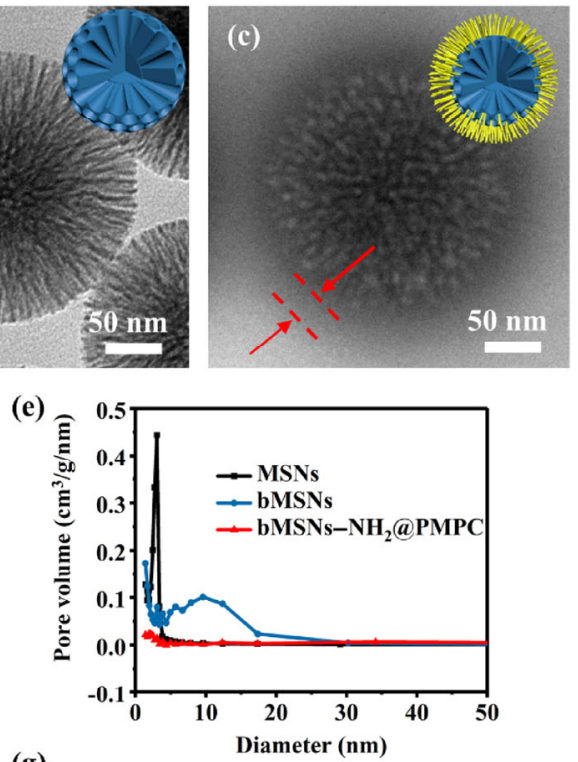

(g)

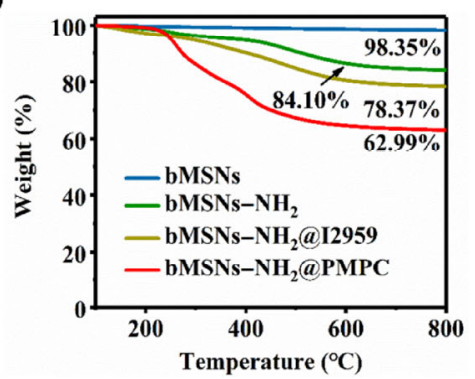

Fig. 2 Characterizations of the nanoparticles. TEM images for (a) MSNs, (b) bMSNs, and (c) bMSNs-NH 2 @PMPC; (d) nitrogen adsorption-desorption isotherms and (e) pore size distribution of MSNs, bMSNs, and bMSNs-NH $\mathrm{NH}_{2}$ PMPC; (f) FTIR spectra and (g) TGA curves of bMSNs, bMSNs- $\mathrm{NH}_{2}$, bMSNs- $\mathrm{NH}_{2} @$ I2959, and bMSNs- $\mathrm{NH}_{2} @$ PMPC.

temperature starting from $100{ }^{\circ} \mathrm{C}$ to eliminate the influence from bound water. It is obvious that the weight loss of bMSNs, bMSNs- $\mathrm{NH}_{2}$, and bMSNs$\mathrm{NH}_{2} @ \mathrm{I} 2959$ is $1.65 \%, 15.90 \%$, and $21.63 \%$, respectively. Therefore, the content of the photoinitiator (I2959-Tos) in bMSNs- $\mathrm{NH}_{2} @ \mathrm{I} 2959$ is calculated to be $\sim 6.81 \%$. The weight loss greatly increases to $37.01 \%$ for bMSNs$\mathrm{NH}_{2} @$ PMPC, and consequently the content of the PMPC polyelectrolyte polymer is calculated to be $\sim 19.24 \%$. The TGA result not only further confirms the successful grafting of the PMPC polyelectrolyte polymer on the nanoparticles surface, but also specifically provides quantitative assessment for each component of the nanoparticles.

The hydrodynamic diameter of the bMSNs, bMSNs$\mathrm{NH}_{2}$, and bMSNs- $\mathrm{NH}_{2} @ \mathrm{I} 2959$ nanoparticles is 289.8, 297.5 , and $280.8 \mathrm{~nm}$, respectively, and there is no significant difference among these nanoparticles. However, the hydrodynamic diameter increases remarkably to $590.3 \mathrm{~nm}$ for bMSNs- $\mathrm{NH}_{2} @ \mathrm{PMPC}$, which is attributed to the hydration effect of the PMPC polyelectrolyte polymer. The zeta potential of bMSNs, bMSNs- $\mathrm{NH}_{2}$, bMSNs- $\mathrm{NH}_{2} @ \mathrm{I} 2959$, and bMSNs$\mathrm{NH}_{2} @ \mathrm{PMPC}$ is $-15.8,37.3,20.3$, and $-5.94 \mathrm{mV}$, respectively. The values of zeta potential for bMSNs and bMSNs- $\mathrm{NH}_{2} @ \mathrm{PMPC}$ are negative because of the presence of the hydroxyl groups and phosphate groups in the nanoparticles, while the positive values of zeta potential for bMSNs- $\mathrm{NH}_{2}$ and bMSNs$\mathrm{NH}_{2} @ \mathrm{I} 2959$ are due to the presence of amino groups.

\subsection{Biodegradation property}

The biodegradation process of the nanoparticles including MSNs, bMSNs, and bMSNs- $\mathrm{NH}_{2} @ \mathrm{PMPC}$ is demonstrated in Fig. 3. It is clearly shown from the TEM images that the morphology of MSNs is not changed from day 0 to day 7 (Fig. 3(a)), indicating that almost no biodegradation has occurred. However, an 

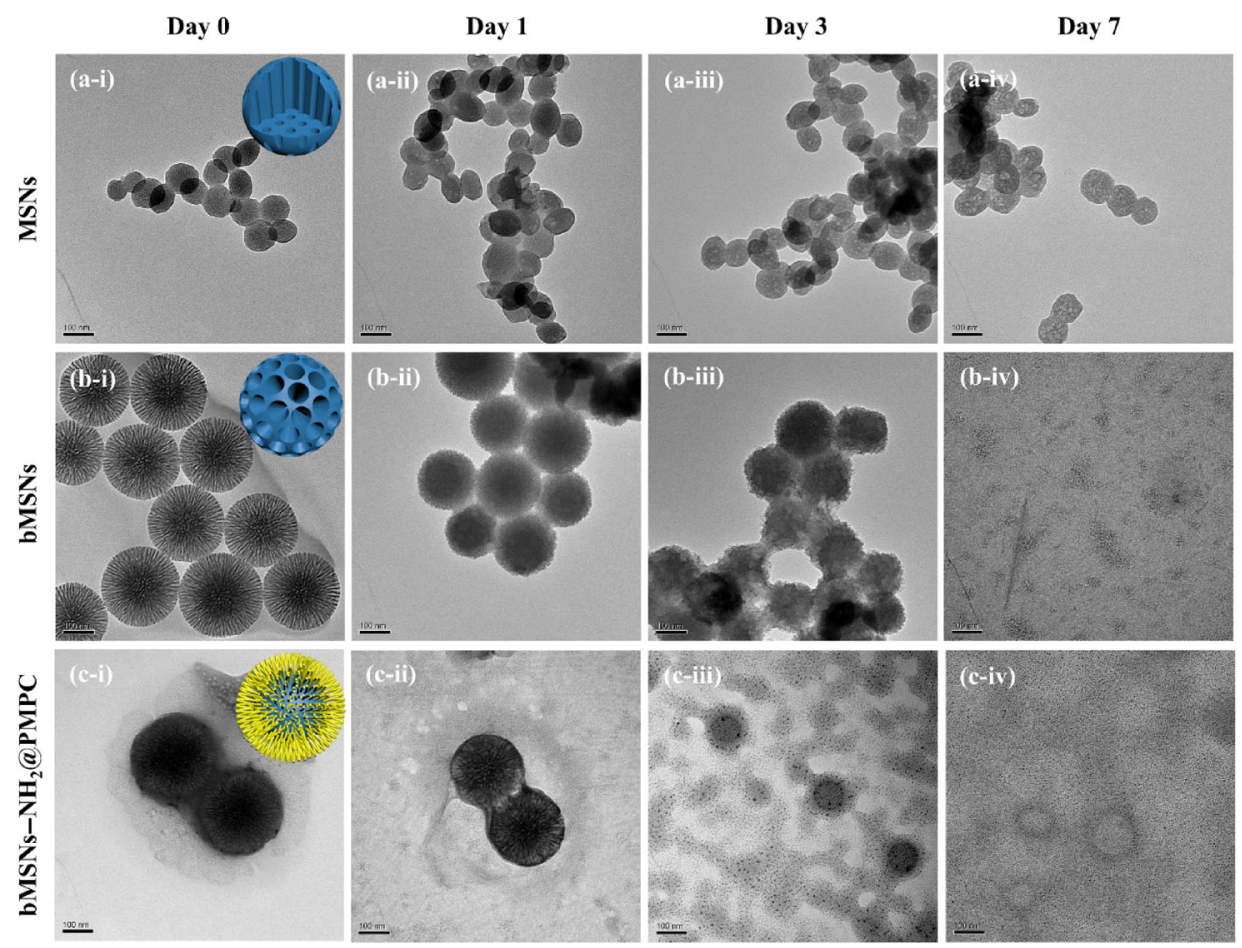

Fig. 3 TEM images showing the biodegradation behavior of the nanoparticles at various time points: (a) MSNs, (b) bMSNs, and (c) bMSNs-NH $\mathrm{NH}_{2} @ \mathrm{PMPC}$.

obvious change has been observed for bMSNs (Fig. 3(b)), as the diameter of the nanoparticles decreases rapidly (i.e., 240, 190, and $140 \mathrm{~nm}$ at day 0,1 , and 3, respectively), and only little remaining residue can be detected at day 7. The same phenomenon is observed for bMSNs- $\mathrm{NH}_{2} @$ PMPC (Fig. 3(c)). The diameter of bMSNs- $\mathrm{NH}_{2} @ \mathrm{PMPC}$ decreases sharply from day 1 to day 3 , and only slight fragments are observed at day 7 .

The faster biodegradation rate of bMSNs and bMSNs- $\mathrm{NH}_{2} @ \mathrm{PMPC}$ is attributed to the lower degree of crosslinking of the silicate backbone and the higher specific surface area. This is caused by the formation of dendritic channels due to mild condensation of the silicate at the oil-water interface in the biphase stratification process [24]. In contrast, the rapid condensing template method [23] for the synthesis of MSNs results in highly crosslinked silicate framework, which can greatly delay the biodegradation process. As a consequence, the biodegradable nanoparticles may be potentially applied in clinics because the toxic effects due to the accumulation can be avoided.

\subsection{Rheological property}

The rheological property of the bMSNs- $\mathrm{NH}_{2} @ \mathrm{PMPC}$ aqueous suspension at different concentrations $(2,4$, 6 , and $10 \mathrm{mg} / \mathrm{mL}$ ) depicted by viscosity versus shear rate curve is shown in Fig. 4(a). Generally, viscosity slightly increases with the increase in shear rate, and it is consistent with the results of a previous study, in which Liu et al. [10] report a shear-thickening behavior of ploy(3-sulfopropyl methacrylate potassium salt)grafted hollow silica nanoparticles in aqueous suspensions. Clinically, hyaluronic acid is used as the viscosupplement to alleviate pain for the patient with osteoarthritis through intra-articular injection to the joint. However, hyaluronic acid has a shear-thinning behavior, and the lubrication property is greatly compromised under higher shear rate [27]. From this viewpoint, the rheological behavior of the bMSNs$\mathrm{NH}_{2} @ \mathrm{PMPC}$, as the lubricant additive, is beneficial for the treatment of osteoarthritis.

\subsection{Lubrication property}

The comparison of COF for the bMSNs and bMSNs$\mathrm{NH}_{2} @ \mathrm{PMPC}$ aqueous suspensions (contact pressure: 19.3 MPa; frequency: $3 \mathrm{~Hz}$; concentration: $5 \mathrm{mg} / \mathrm{mL}$ ) is displayed in Fig. 4(b), and the schematic diagram of the tribological test is shown in Fig. 4(c). The COF 


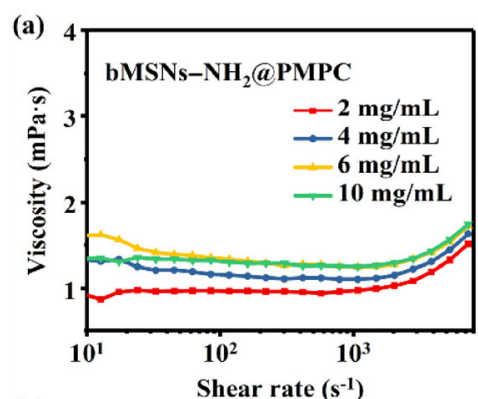

(b)

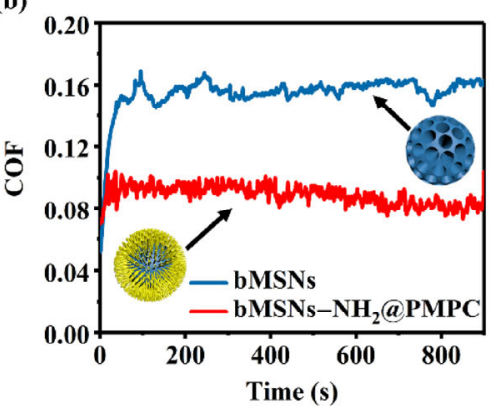

(c)

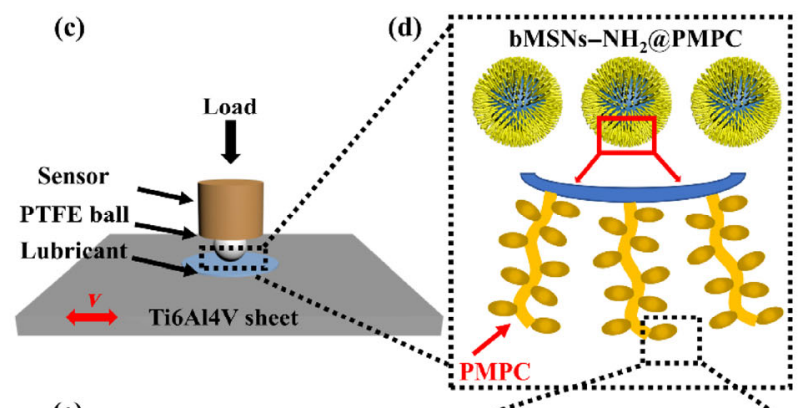

(e)

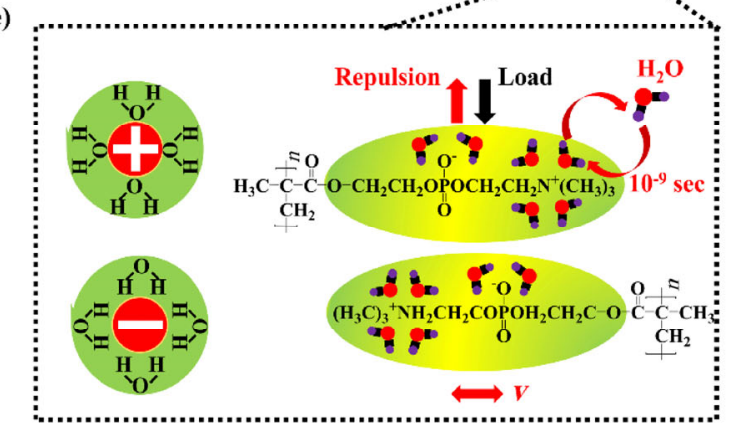

Fig. 4 Rheological and lubrication properties of the nanoparticles: (a) viscosity-shear rate curves of bMSNs-NH $\mathrm{N}_{2}$ PMPC at various concentrations; (b) COF-time plots of bMSNs and bMSNs-NH $\mathrm{N}_{2}$ (PMPC; (c) schematic diagram of the tribological test setup; (d, e) schematic illustration showing the hydration lubrication mechanism based on the formation of hydration layer surrounding the phosphocholine headgroups in bMSNs- $\mathrm{NH}_{2} @$ PMPC.

of bMSNs- $\mathrm{NH}_{2} @ \mathrm{PMPC}$ is 0.088 , which is much lower than that of bMSNs (0.158). It is considered that the reduction in $\mathrm{COF}$ is attributed to hydration lubrication mechanism of the PMPC polyelectrolyte polymer grafted on the surface of bMSNs nanoparticles [28]. The zwitterionic phosphocholine headgroups in PMPC is the same as that of the phosphatidylcholine lipid in articular cartilage, which lubricates the joint based on the same mechanism [29, 30]. As demonstrated in Figs. 4(d) and 4(e), the water molecules can form a tenacious hydration layer around the zwitterionic $\mathrm{N}^{+}\left(\mathrm{CH}_{3}\right)_{3}$ and $\mathrm{PO}_{4}^{-}$charges in the bMSNs- $\mathrm{NH}_{2} @ \mathrm{PMPC}$ due to the interaction between water dipole and enclosed charges. The hydration layer can support high pressure without deformation because of the steric effect, and respond in a fluidlike manner under shear with a high speed exchange between the water molecules in the hydration layer and the nearby "free" water molecules (typically the time scale in the order of $10^{-9} \mathrm{~s}$ ). Consequently, it results in a great reduction in the interfacial friction of the contact tribopair.

The surface morphology of the wear areas on the Ti6Al4V sheet using the bMSNs and bMSNs$\mathrm{NH}_{2} @ \mathrm{PMPC}$ aqueous suspensions as the lubricant is shown in Fig. 5. With regard to bMSNs, the trace of wear area is slightly visible and partly covered by some irregular fragments (Fig. 5(a)). The elemental distribution of the fragments corresponds to silicon, as displayed in Fig. 5(b). This result indicates that the bMSNs nanoparticles adsorb onto the Ti6Al4V sheet during the tribological test, which may cause abrasive wear of the tribopair. Likewise, a layer of bMSNs$\mathrm{NH}_{2} @$ PMPC nanoparticles is also detected on the Ti6Al4V sheet, and the trace of wear area on the Ti6Al4V sheet is hardly observed, as shown in Figs. 5(c) and $5(\mathrm{~d})$. The presence of the bMSNs- $\mathrm{NH}_{2} @ \mathrm{PMPC}$ nanoparticles can effectively reduce wear generation in the tribological test.

The comparison of lubrication properties of the bMSNs and bMSNs- $\mathrm{NH}_{2} @ \mathrm{PMPC}$ aqueous suspensions under different experimental conditions are demonstrated in Fig. 6. Figures 6(a) and 6(b) present the COF-time plots of bMSNs and bMSNs- $\mathrm{NH}_{2} @ \mathrm{PMPC}$ under various loads of 1,2 , and $4 \mathrm{~N}$ (frequency: $3 \mathrm{~Hz}$ ). The COF remains stable during the test, and the average value is much lower for bMSNs- $\mathrm{NH}_{2} @ \mathrm{PMPC}$ $(1 \mathrm{~N}: 0.107 ; 2 \mathrm{~N}: 0.089 ; 5 \mathrm{~N}: 0.082)$ than that of bMSNs $(1 \mathrm{~N}: 0.162 ; 2 \mathrm{~N}: 0.155 ; 5 \mathrm{~N}: 0.152)$. Figures 6(c) and 6(d) illustrate the COF-time plots of bMSNs and 


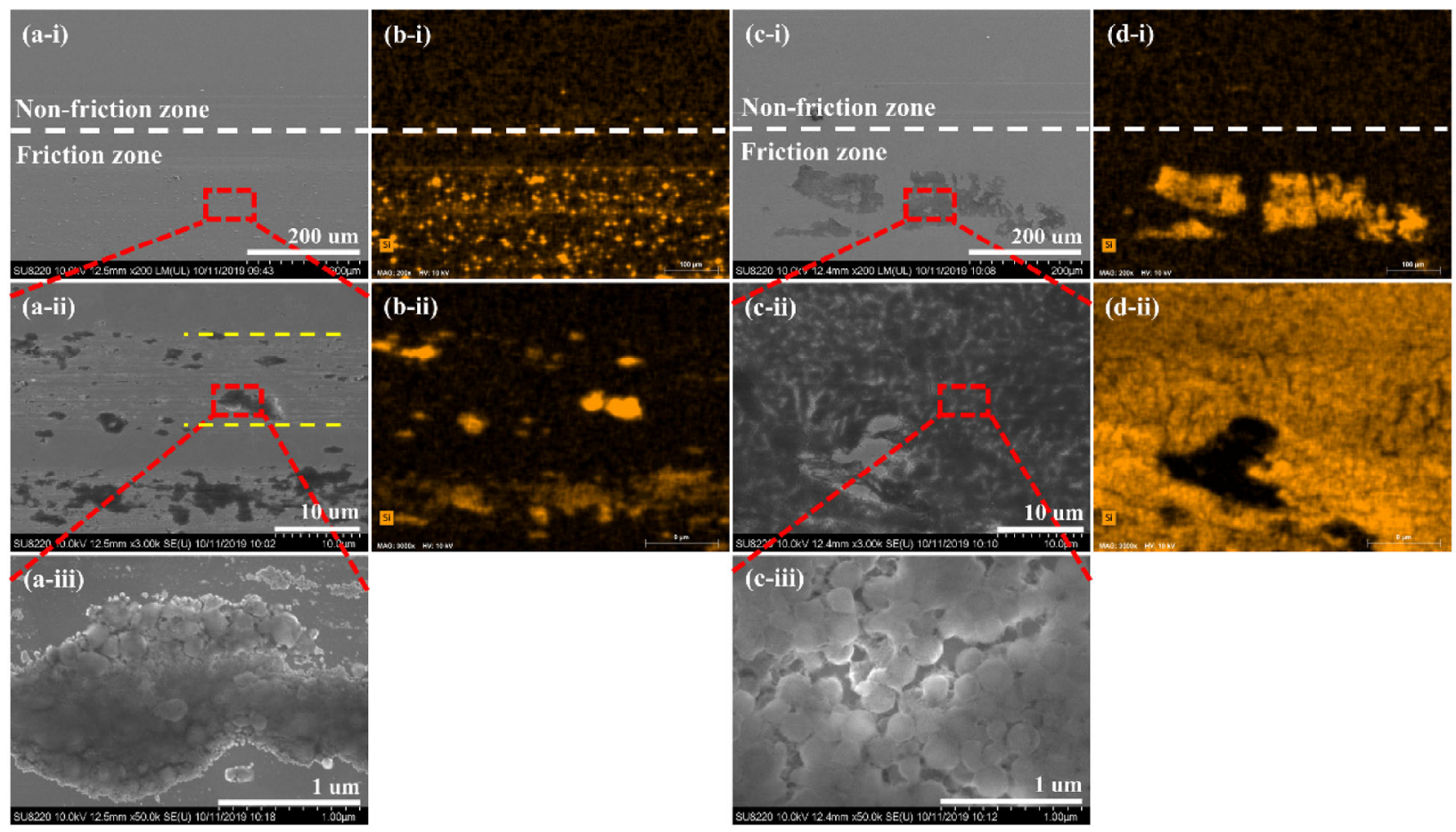

Fig. 5 Surface morphology and elemental distribution of the wear areas using bMSNs and bMSNs- $\mathrm{NH}_{2} @ \mathrm{PMPC}$ as the lubricant: (a, b) bMSNs and (c, d) bMSNs-NH $@$ PMPC.
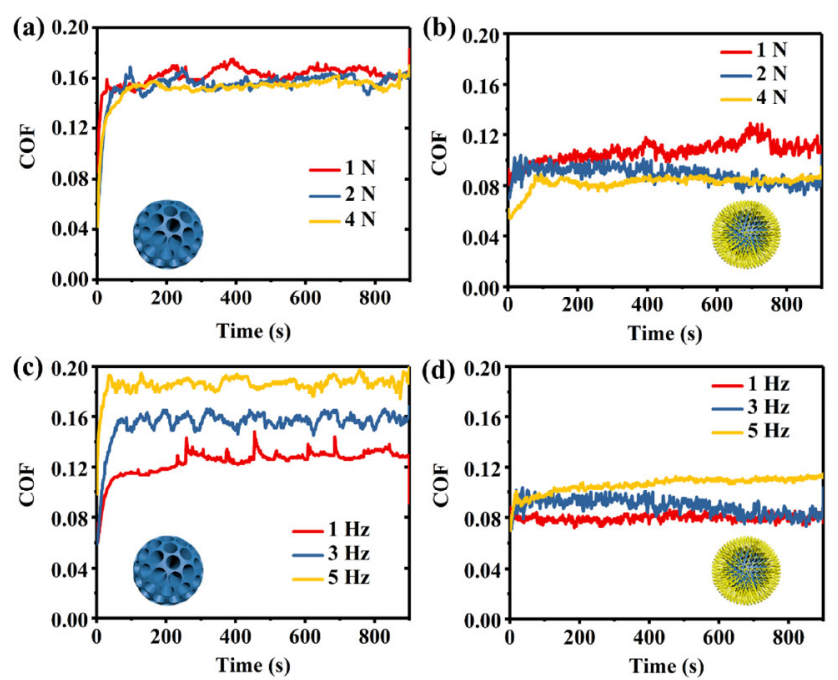

Fig. 6 Lubrication property of the nanoparticles under different experimental conditions: COF-time plots of (a) bMSNs and (b) bMSNs- $\mathrm{NH}_{2} @ \mathrm{PMPC}$ under various loads of 1, 2, and $4 \mathrm{~N}$; COF-time plots of (c) bMSNs and (d) bMSNs-NH $\mathrm{NH}_{2} @ \mathrm{PMPC}$ under various frequencies of 1,3 , and $5 \mathrm{~Hz}$.

bMSNs- $\mathrm{NH}_{2} @ \mathrm{PMPC}$ under various frequencies of 1, 3, and $5 \mathrm{~Hz}$ (load: $2 \mathrm{~N}$ ). Similarly, the average value of COF for bMSNs-NH $\mathrm{N}_{2} @$ PMPC (1 Hz: 0.080; $3 \mathrm{~Hz}$ : 0.088 ; $5 \mathrm{~Hz}: 0.106)$ is much lower than that of bMSNs (1 Hz: 0.124; $3 \mathrm{~Hz}$ : 0.155; $5 \mathrm{~Hz}$ : 0.186). As previously mentioned, the load employed in the tribological test corresponds equivalently to the maximum contact pressure of $15.4 \mathrm{MPa}(1 \mathrm{~N}), 19.3 \mathrm{MPa}(2 \mathrm{~N})$, and $24.4 \mathrm{MPa}(4 \mathrm{~N})$, respectively, which is larger than the typical pressure at the human joint ( 5 MPa) [31, 32].

The result of lubrication property of biodegraded bMSNs and bMSNs- $\mathrm{NH}_{2} @ \mathrm{PMPC}$ in aqueous suspensions is displayed in Figs. 7(a) and 7(b). Generally, the COF values of the two nanoparticles decrease with the biodegradation time from day 1 to day 7 . With regard to bMSNs, the COF shows a rapid decrease on day 5 compared with that of day 3 (Fig. 7(a)), while it is slightly changed for other time points. However, the COF of bMSNs- $\mathrm{NH}_{2} @ \mathrm{PMPC}$ presents a gradually decreasing trend for all the tested time points. Combining with previous TEM images of the biodegraded nanoparticles, it is indicated that the bMSNs- $\mathrm{NH}_{2} @$ PMPC keep an excellent lubrication property during the biodegradation process.

\subsection{In vitro drug release}

The LC of the MSNs, bMSNs, and bMSNs- $\mathrm{NH}_{2} @ \mathrm{PMPC}$ nanoparticles is calculated to be $5.69 \%, 10.5 \%$, and $1.22 \%$, respectively. As expected, the value decreases 


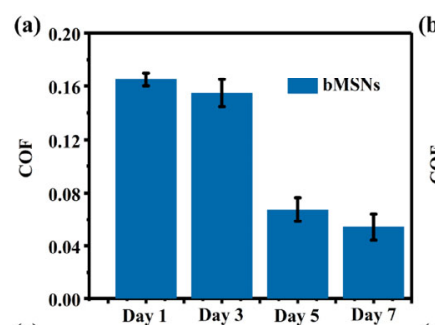

(c)
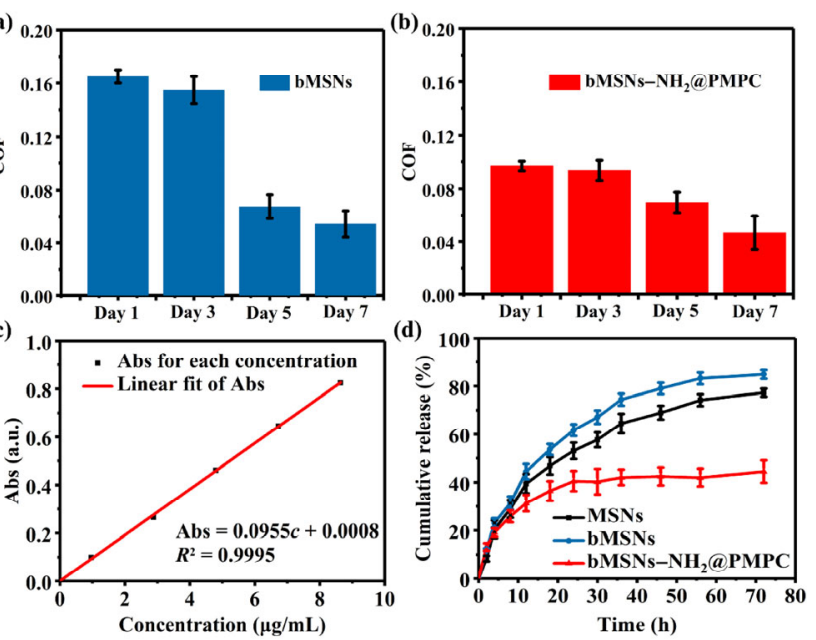

Fig. 7 Lubrication property of the biodegraded nanoparticles for (a) bMSNs and (b) bMSNs- $\mathrm{NH}_{2} @ \mathrm{PMPC}$; (c) calibration curve of RhB with different concentrations; (d) drug release profiles of the MSNs, bMSNs, and bMSNs- $\mathrm{NH}_{2} @$ PMPC. Abs: absorbance.

greatly following grafting of PMPC polyelectrolyte polymer on the bMSNs surface. Likewise, the EE of the MSNs, bMSNs, and bMSNs- $\mathrm{NH}_{2} @ \mathrm{PMPC}$ nanoparticles is $47.3 \%, 91.6 \%$, and $9.67 \%$, respectively. The calibration curve of the model drug RhB in PBS is shown in Fig. 7(c), and the cumulative drug release profiles of the RhB-loaded MSNs, bMSNs, and bMSNs- $\mathrm{NH}_{2} @ \mathrm{PMPC}$ are demonstrated in Fig. 7(d). At 72 h, $45.8 \%$ of RhB is released from bMSNs- $\mathrm{NH}_{2} @ \mathrm{PMPC}$, which is much lower than that from MSNs (78.1\%) and bMSNs (86.3\%). It is considered that the PMPC polyelectrolyte polymer grafted on the bMSNs surface results in sustained drug release behavior of the bMSNs- $\mathrm{NH}_{2} @ \mathrm{PMPC}$.

\section{Conclusions}

In this study, bMSNs- $\mathrm{NH}_{2} @ \mathrm{PMPC}$ with enhanced lubrication and sustained drug release properties were synthesized via photopolymerization. The nanoparticles were characterized by TEM, BET, FTIR, and TGA to confirm the successful grafting of the PMPC polyelectrolyte polymer on the bMSNs surface. The biodegradation test demonstrated that the bMSNs$\mathrm{NH}_{2} @$ PMPC almost degraded completely in SBF within 7 days, thus avoiding the potential toxic effect due to accumulation in vivo. The lubrication test showed improved lubrication property of the bMSNs$\mathrm{NH}_{2} @$ PMPC (compared with the bMSNs) under different experimental conditions. This was attributed to the hydration lubrication mechanism of the zwitterionic phosphocholine headgroups in PMPC. Importantly, the bMSNs- $\mathrm{NH}_{2} @ \mathrm{PMPC}$ under biodegradation maintained stable lubrication performance. Additionally, the drug release test indicated the bMSNs- $\mathrm{NH}_{2} @$ PMPC could release the pre-loaded drug with a sustained behavior. Consequently, the bMSNs- $\mathrm{NH}_{2} @ \mathrm{PMPC}$ developed herein may be used for biomedical applications to treat such diseases where lubrication enhancement and drug intervention are specifically required, i.e., early stage osteoarthritis.

\section{Acknowledgements}

This study was financially supported by National Natural Science Foundation of China (51675296), Tsinghua University-Peking Union Medical College Hospital Initiative Scientific Research Program (20191080593), Tsinghua University Initiative Scientific Research Program (20197050026), Foshan-Tsinghua Innovation Special Fund (FTISF), Research Fund of State Key Laboratory of Tribology, Tsinghua University, China (SKLT2020C11), and Ng Teng Fong Charitable Foundation (202-276-132-13).

Open Access This article is licensed under a Creative Commons Attribution 4.0 International License, which permits use, sharing, adaptation, distribution and reproduction in any medium or format, as long as you give appropriate credit to the original author(s) and the source, provide a link to the Creative Commons licence, and indicate if changes were made.

The images or other third party material in this article are included in the article's Creative Commons licence, unless indicated otherwise in a credit line to the material. If material is not included in the article's Creative Commons licence and your intended use is not permitted by statutory regulation or exceeds the permitted use, you will need to obtain permission directly from the copyright holder.

To view a copy of this licence, visit http://creativecommons.org/licenses/by/4.0/.

\section{References}

[1] Jin Z M, Dowson D. Bio-friction. Friction 1(2): 100-113 (2013) 
[2] Glyn-Jones S, Palmer A J R, Agricola R, Price A J, Vincent T L, Weinans H, Carr A J. Osteoarthritis. Lancet 386(3991): 376-387 (2015)

[3] Halilaj E, Le Y, Hicks J L, Hastie T J, Delp S L. Modeling and predicting osteoarthritis progression: Data from the osteoarthritis initiative. Osteoart Cartil 26(12): 1643-1650 (2018)

[4] Wimmer M A, Birken L, Sellenschloh K, Schneider E. Damage due to rolling in total knee replacement-the influence of tractive force. Friction 1(2): 178-185 (2013)

[5] Ji X L, Zhang H Y. Current strategies for the treatment of early stage osteoarthritis. Front Mech Eng 5: 57 (2019)

[6] Choi Y J, Kim S O, Sim J H, Hahm K D. Postoperative anemia is associated with acute kidney injury in patients undergoing total hip replacement arthroplasty: A retrospective study. Anesth Analg 122(6): 1923-1928 (2016)

[7] Podmore B, Hutchings A, Konan S, van der Meulen J. The agreement between chronic diseases reported by patients and derived from administrative data in patients undergoing joint arthroplasty. BMC Med Res Methodol 19(1): 87 (2019)

[8] Ji X L, Yan Y F, Sun T, Zhang Q, Wang Y X, Zhang M, Zhang H Y, Zhao X. Glucosamine sulphate-loaded distearoyl phosphocholine liposomes for osteoarthritis treatment: Combination of sustained drug release and improved lubrication. Biomater Sci 7(7): 2716-2728 (2019)

[9] Zheng Y W, Yang J L, Liang J, Xu X Y, Cui W G, Deng L F, Zhang H Y. Bioinspired hyaluronic acid/phosphorylcholine polymer with enhanced lubrication and anti-inflammation. Biomacromolecules 20(11): 4135-4142 (2019)

[10] Liu G Q, Cai M R, Zhou F, Liu W M. Charged polymer brushes-grafted hollow silica nanoparticles as a novel promising material for simultaneous joint lubrication and treatment. $J$ Phys Chem B 118(18): 4920-4931 (2014)

[11] Yan Y F, Sun T, Zhang H B, Ji X L, Sun Y L, Zhao X, Deng L F, Qi J, Cui W G, Santos H A, et al. Euryale ferox seed-inspired superlubricated nanoparticles for treatment of osteoarthritis. Adv Funct Mater 29(4): 1807559 (2019)

[12] Tan X L, Sun Y L, Sun T, Zhang H Y. Mechanised lubricating silica nanoparticles for on-command cargo release on simulated surfaces of joint cavities. Chem Commun 55(18): 2593-2596 (2019)

[13] Shao D, Lu M M, Zhao Y W, Zhang F, Tan Y F, Zheng X, Pan Y, Xiao X A, Wang Z, Dong W F, et al. The shape effect of magnetic mesoporous silica nanoparticles on endocytosis, biocompatibility and biodistribution. Acta Biomater 49: 531-540 (2017)

[14] Wei Y C, Quan L, Zhou C, Zhan Q Q. Factors relating to the biodistribution \& clearance of nanoparticles \& their effects on in vivo application. Nanomedicine 13(12): 1495-1512 (2018)

[15] Pohaku Mitchell K K, Liberman A, Kummel A C, Trogler W C. Iron (III)-doped, silica nanoshells: A biodegradable form of silica. J Am Chem Soc 134(34): 13997-14003 (2012)

[16] Kim I Y, Joachim E, Choi H, Kim K. Toxicity of silica nanoparticles depends on size, dose, and cell type. Nanomed Nanotechnol Biol Med 11(16): 1407-1416 (2015)

[17] Fu C H, Liu T L, Li L L, Liu H Y, Chen D, Tang F Q. The absorption, distribution, excretion and toxicity of mesoporous silica nanoparticles in mice following different exposure routes. Biomaterials 34(10): 2565-2575 (2013)

[18] Goel S, Chen F, Luan S J, Valdovinos H F, Shi S X, Graves S A, Ai F R, Barnhart T E, Theuer C P, Cai W B. Engineering intrinsically zirconium-89 radiolabeled self-destructing mesoporous silica nanostructures for in vivo biodistribution and tumor targeting studies. Adv Sci 3(11): 1600122 (2016)

[19] Du X, Li X Y, Xiong L, Zhang X J, Kleitz F, Qiao S Z. Mesoporous silica nanoparticles with organo-bridged silsesquioxane framework as innovative platforms for bioimaging and therapeutic agent delivery. Biomaterials $\mathbf{9 1}$ : 90-127 (2016)

[20] Shao D, Li M Q, Wang Z, Zheng X, Lao Y H, Chang Z M, Zhang F, Lu M M, Yue J, Hu H Z, et al. Bioinspired diselenide-bridged mesoporous silica nanoparticles for dualresponsive protein delivery. Adv Mater 30(29): 1801198 (2018)

[21] Li H M, Guo H L, Lei C, Liu L, Xu L Q, Feng Y P, Ke J, Fang W, Song H, Xu C, et al. Nanotherapy in joints: Increasing endogenous hyaluronan production by delivering hyaluronan synthase 2. Adv Mater 31(46): 1904535 (2019)

[22] Wang Z, Zhang F, Shao D, Chang Z M, Wang L, Hu H Z, Zheng X, Li X Z, Chen F M, Tu Z X, et al. Janus nanobullets combine photodynamic therapy and magnetic hyperthermia to potentiate synergetic anti-metastatic immunotherapy. $A d v$ Sci 6(22): 1901690 (2019)

[23] Huh S, Wiench J W, Yoo J C, Pruski M, Lin V S Y. Organic functionalization and morphology control of mesoporous silicas via a co-condensation synthesis method. Chem Mat 15(22): 4247-4256 (2003)

[24] Shen D K, Yang J P, Li X M, Zhou L, Zhang R Y, Li W, Chen L, Wang R, Zhang F, Zhao D Y. Biphase stratification approach to three-dimensional dendritic biodegradable mesoporous silica nanospheres. Nano Lett 14(2): 923-932 (2014)

[25] Liu S Z, Zhang Q, Han Y, Sun Y L, Zhang Y F, Zhang H Y. Bioinspired surface functionalization of titanium alloy for enhanced lubrication and bacterial resistance. Langmuir 
35(40): 13189-13195 (2019)

[26] Zhang H Y, Zhang S H, Luo J B, Liu Y H, Qian S H, Liang F H, Huang Y L. Investigation of protein adsorption mechanism and biotribological properties at simulated stem-cement interface. J Tribol 135(3): 032301 (2013)

[27] Sundaram H, Voigts B, Beer K, Meland M. Comparison of the rheological properties of viscosity and elasticity in two categories of soft tissue fillers: Calcium hydroxylapatite and hyaluronic acid. Dermatol Surg 36(S3): 1859-1865 (2010)

[28] Klein J. Hydration lubrication. Friction 1(1): 1-23 (2013)

[29] Wang Y X, Sun Y L, Gu Y H, Zhang H Y. Articular

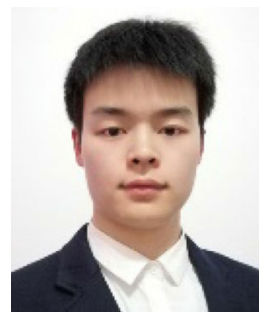

Li WAN. He received his B.S. in applied chemistry in 2017 from Hunan University of Arts and Science, China. He is currently a

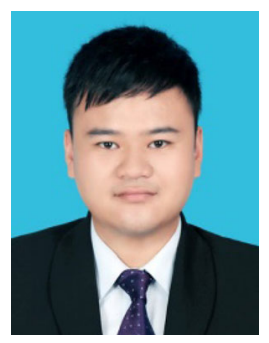

Yi WANG. He received his B.S. in materials science \& engineering in 2013 from Hainan University, China. Then he received his M.S. degree in biomedical engineering in 2016

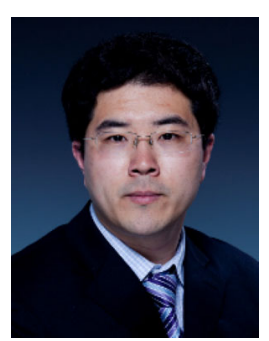

Hongyu ZHANG. He received his B.S. in Tianjin University, China (2005) and Ph.D. in University of Huddersfield, UK (2009). He is an associate professor at the State Key Laboratory of Tribology, Department of Mechanical Engineering, Tsinghua University, China. His research interests focus on the cartilage-inspired surface functionalization for enhanced lubrication. Adv Mater Interfaces 6(12): 1900180 (2019)

[30] Murakami T, Yarimitsu S, Nakashima K, Sawae Y, Sakai N. Influence of synovia constituents on tribological behaviors of articular cartilage. Friction 1(2): 150-162 (2013)

[31] Klein J. Repair or replacement-a joint perspective. Science 323(5910): 47-48 (2009)

[32] Han Y, Liu S Z, Sun Y L, Gu Y H, Zhang H Y. Bioinspired surface functionalization of titanium for enhanced lubrication and sustained drug release. Langmuir 35(20): 6735-6741 (2019)

joint master student in Tsinghua University, China. His research interests include nanomaterials, biotribology, and drug delivery.

from Southwest Jiaotong University, China. After that he was a Ph.D. candidate in mechanical engineering in Tsinghua University, China. His research interests include bio-tribology, bio-fabrication, and nano-sensors.

development of lubricating biomaterials such as nanoparticles, coatings, hydrogels, and electrospun nanofibers for biomedical applications, which integrate the multi-disciplinary knowledge including biotribology, chemistry, materials science, and medicine to address clinical issues, e.g., osteoarthritis, anti-tissue/cell/bacteria adhesion, bone tissue engineering, etc. 